\title{
FACTORS RELATED TO THE FINAL VISUAL OUTCOME OF 425 PATIENTS WITH TRAUMATIC HYPHAEMA
}

\author{
C. S. NG, J.M. SPARROW, N. P. STRONG and A. R. ROSENTHAL \\ Leicester
}

\begin{abstract}
SUMMARY
A retrospective study of the visual outcome of 425 inpatients with traumatic hyphaema has been conducted. A multivariate analysis demonstrated that after adjusting for age, sex and pre-existing poor vision, the size of hyphaema on presentation and the presence of retinal damage were significant predictors of a worse final visual outcome ( $p=0.00003$ and 0.00001 respectively). Topical steroid and/or cycloplegic medication, and the occurrence of secondary haemorrhage did not influence the final visual outcome after adjustment for the other variables. These data illustrate, in an unselected sequential population of patients, the role of these factors in terms of final visual outcome following hyphaema from blunt ocular trauma.
\end{abstract}

Traumatic hyphaema due to blunt injury is associated with an increased risk of compromised visual function. The causes of poor vision after blunt injury include retinal commotio and retinal detachment, anterior chamber angle damage with glaucoma damage to the crystalline lens, and damage to other ocular structures. ${ }^{1-12}$ In addition, the primary hyphaema or a secondary haemorrhage may result in corneal blood staining. ${ }^{8-12}$ The relative importance of these factors in determining the final visual outcome after traumatic hyphaema has not been well. established. Although some of these factors, such as retinal commotio, are not amenable to treatment, others, such as retinal detachment or secondary haemorrhage may respond to treatment.

In order to determine the relative importance of these factors we have performed a study in which we have assessed the final visual outcome following traumatic hyphaema. Factors pertinent to the injury and the type of treatment employed were examined to determine the risk factors for poor visual outcome.

\section{PATIENTS AND METHODS}

The hospital records of in-patients admitted for blunt

From: Department of Ophthalmology, Leicester Royal Infirmary.

Correspondence to: Dr C S Ng, Department of Ophthalmology, Leicester Royal Infirmary, Leicester LE1 5WW. ocular trauma with hyphaema were identified and reviewed. The details of this patient population have been described. ${ }^{13}$ Data on the final visual acuity of the eyes following injury were available for 425 of these individuals.

The final visual outcome was defined as the corrected visual acuity (spectacles or pin-hole) recorded at the last attendance of the patient. For patients who failed to return for follow-up, the final visual acuity was taken as the visual acuity recorded on the day of discharge from the ward. If this information was unavailable, then the patient was excluded from this study. The reason for poor visual outcome $(6 / 18$ or worse) was recorded when that information was available.

The data collected on each patient included age, sex, the nature of the injury, the existence of poor vision due to preexisting ocular pathology, the grade of initial hyphaema, intra-ocular pressure on presentation, treatment employed, the presence of complications (including secondary haemorrhage and retinal injuries), ${ }^{13}$ the final visual outcome and miscellaneous information relevant to individual cases.

The method used for hyphaema grading has been described, ${ }^{13}$ and secondary haemorrhage was defined as having occurred when there was fresh layering of blood in the anterior chamber on slit lamp examination.

\section{STATISTICAL METHODS}

A sequential multivariate analysis was performed to determine risk factors related to the injury, and to assess the effects of treatment on visual outcome. The main outcome variable was the final visual acuity. Visual acuity was modelled as the log of the reciprocal of the Snellen fraction using a combined multiple regression and factor analysis. In all analyses, prior adjustment for age, sex and pre-existing poor vision was made, and when examining the effect of an individual complication, the effects of other complications were treated as confounding variables. The analysis was performed using the Generalised Linear Interactive Modelling system (GLIM 3.77).*

*GLIM Numerical Algorithms Group Ltd. (NAG), Wilkinson House, Jordon Hill Road, Oxford, OX2 8DR U.K. 
FIG. 1: DISTRIBUTION OF FINAL VISUAL ACUITY

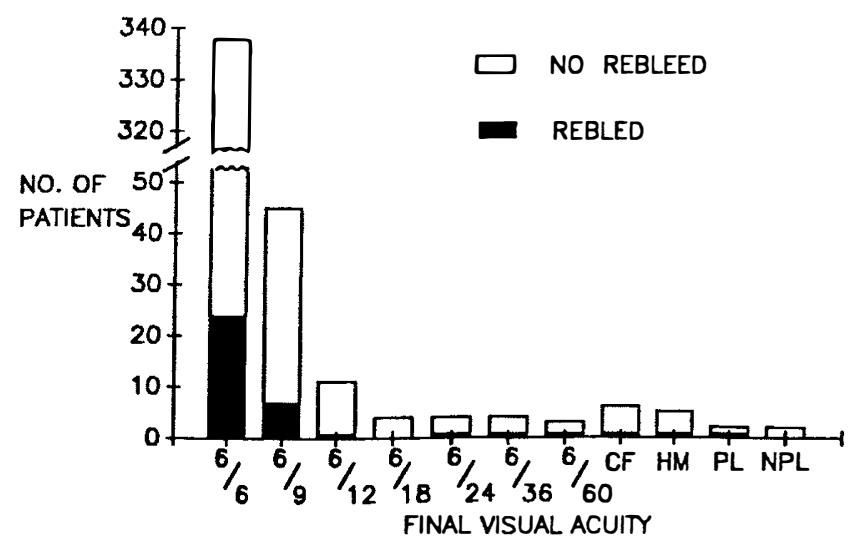

Fig. 1. Distribution of the final Snellen visual acuity following blunt ocular trauma. Also indicated (filled bar sections) are those individuals who suffered secondary haemorrhage.

\section{RESULTS}

The distribution of the final visual acuity is presented in Figure 1 . Overall $7 \%(30 / 425)$ of eyes had a final visual acuity of $6 / 18$ or worse. Visual outcome by retinal involvement, secondary haemorrhage and size of initial hyphaema is presented in Table I, where visual outcome is divided into those with a "good" outcome $(6 / 12$ or better), and those with a "poor" outcome (6/18 or worse). The causes of the visual reduction in patients with a final visual acuity of $6 / 18$ or worse are listed in Table II. It is noteworthy that pre-existing pathology and retinal damage associated with the blunt injury accounted for most of the visual morbidity.

In the multrivariate analysis, after adjusting for age, sex and pre-existing poor vision, it was found that a higher grade of hyphaema on presentation $(\mathrm{p}=0.00003)$ and the presence of retinal damage $(\mathrm{p}=0.00001)$ were each associated with worse final visual outcome. Treatment with topical steroid and/or cycloplegics, however, had no significant effect on final visual outcome.

A separate analysis was performed to determine whether there was any association between pre-existing poor vision and hyphaema size, retinal damage, and secondary haemorrhage. After adjustment for age and sex, there was no significant association between pre-existing poor vision and these three factors. A further analysis

Table I. Visual outcome of patients with and without retinal involvement, rebleeding, and by grade of initial hyphaema

\begin{tabular}{lcc}
\hline & $\begin{array}{r}\text { VA 6/12 or better } \\
\text { (Row \%) }\end{array}$ & $\begin{array}{c}\text { VA 6/18 or worse } \\
\text { (Row \%) }\end{array}$ \\
\hline Retina involved & $57(80 \%)$ & $14(20 \%)$ \\
Retina not involved & $338(95 \%)$ & $16(5 \%)$ \\
Rebleed & $32(84 \%)$ & $6(16 \%)$ \\
No rebleed & $363(94 \%)$ & $24(6 \%)$ \\
Grade 0 & $58(92 \%)$ & $5(8 \%)$ \\
Grade 1 & $233(94 \%)$ & $14(6 \%)$ \\
Grade 2 & $83(94 \%)$ & $5(6 \%)$ \\
Grade 3 & $12(80 \%)$ & $3(20 \%)$ \\
Grade 4 & $9(75 \%)$ & $3(25 \%)$ \\
Overall & $395(93 \%)$ & $30(7 \%)$ \\
\hline
\end{tabular}

examined whether the injured eye was more likely to have had previous poor vision than the uninjured eye. No significant association was found, indicating that patients with previous poor vision in one eye were no more likely to have sustained an injury in their poorly sighted eye than in their fellow eye.

\section{DISCUSSION}

After accounting for confounding variables, our data indicate that the final visual outcome of patients who had a larger hyphaema was significantly poorer $(\mathrm{p}=0.00003)$. This is an agreement with many series that have reported significant correlations between hyphaema size and visual outcome. ${ }^{7}$, 10, 14-17

Our study does not, however, support previous reports that secondary haemorrhage is associated with a poorer visual outcome. ${ }^{3,4,6,8,10,17,18}$ It is self evident that retinal damage and pre-existing poor vision would have an impact upon final visual outcome following blunt ocular trauma. In previous studies prior adjustment has not been made for these important confounding factors, and indeed in our study we found a weak but significant effect of secondary haemorrhage in the preliminary analysis, which disappeared when the confounding variables were taken into account in the multivariate analysis. This suggests that the validity of the findings of previous studies with regard to the importance of secondary haemorrhage and visual outcome should be questioned. This is not to say that secondary haemorrhage may not occasionally, in individual cases, lead to visual loss, but in our series of 425 patients this was not an important cause of visual morbidity.

Almost all the patients who had a visual outcome of $6 / 18$ or worse had incurred retinal damage or had preexisting pathology (Table II). A single exceptional patient with severe idiopathic thrombocytopaenia had multiple secondary haemorrhages, with attendant raised intraocular pressure and corneal blood staining. His final visual acuity was hand movements due to glaucoma and corneal blood staining despite surgical evacuation of the blood clot and cataract extraction. This patient had previously refused a splenectomy which had been recommended as essential to the management of his thrombocytopaenia. One other patient required surgical evacuation of a clot following a secondardy haemorrhage which failed to absorb and which caused acute secondary glaucoma which could not be controlled medically. The final visual acuity of this patient was $6 / 6$.

Table II. Reasons for poor final visual outcome

\begin{tabular}{|c|c|c|c|c|c|c|c|c|}
\hline & $6 / 18$ & $6 / 24$ & $6 / 36$ & $6 / 60$ & $\mathrm{CF}$ & $\mathrm{HM}$ & PL & NPL \\
\hline $\begin{array}{l}\text { Pre-existing } \\
\text { pathology }\end{array}$ & & 2 & 2 & 2 & 2 & 2 & 1 & 1 \\
\hline Retinal injury & 3 & 2 & 2 & 1 & 3 & 2 & 1 & 1 \\
\hline $\begin{array}{l}\text { Complications of } \\
\text { rebleed } \\
\text { Unknown }\end{array}$ & 1 & & & & 1 & 1 & & \\
\hline
\end{tabular}

$\mathrm{CF}=$ counting fingers; $\mathrm{HM}=$ hand movement; $\mathrm{PL}=$ perception of light; $\mathrm{NPL}=$ no perception of light. 
It is of interest in this series that of the 30 eyes with a final visual outcome of $6 / 18$ or worse, $12(40 \%)$ of these had poor vision prior to the blunt injury (Table I). If we exclude these 12 eyes, then $96 \%$ (395/413) of the remaining eyes had a final acuity of $6 / 12$ or better following the blunt injury.

We conclude from our data that following blunt ocular trauma the characteristics of the hyphaema are related to the final visual outcome, with larger haemorrhages representing a greater risk for poor vision. Retinal damage is confirmed as an important contributor to a worse final visual outcome, although at the time of the initial examination such damage is frequently not visible through the hyphaema. Our study has failed to demonstrate any important effect of secondardy haemorrhage on final visual outcome following blunt ocular trauma.

Key words: Visual outcome - Hyphaema retinal injury secondary Haemorrhage.

\section{REFERENCES}

1. Eagling EM: Ocular damage after blunt trauma to the eye. Br J Ophthalmol 1974, 58: 126-40.

2. Kearns P: Traumatic hyphaema: a retrospective study of 314 cases: Br J Ophthalmol 1991, 75: 137-41.

3. Gregersen E: Traumatic hyphaema. Acta Ophthalmol 1962, 40: 192-9.

4. Agapitos PJ, Noel L-P, Clarke WN: Traumatic hyphaema in children. Ophthalmology 1987, 94: 1238-41.

5. Uusitalo RJ, Ranta-Kemppainen L, Tarkkanen A: Manage- ment of traumatic hyphema in children. An analysis of 340 cases. Arch Ophthalmol 1988, 106: 1207-9.

6. Loring MJ: Traumatic hyphema. Am J Ophthalmol 1958, 46: $873-880$.

7. Oksala A: Treatment of traumatic hyphaema. Acta Ophthalmol 1967, 51: 315-20.

8. Shea M: Traumatic hyphaema in children. A review of 113 cases: Canad Med Ann J 1957, 76: 466-9.

9. Spoor TC, Kwitko GM, O'Grady JM, Ramocki JM: Traumatic hyphema in an urban population. Am J Ophthalmol 1990, 109: 23-7.

10. Thygeson $\mathrm{P}$, and Beard $\mathrm{C}$ : Observations on traumatic hyphema. Am J Ophthalmol 1952, 35: 977-85.

11. Gilbert HD and Jensen AD: Atropine in the treatment of traumatic hyphema. Ann Ophthalmol 1973, 5: 1297-300.

12. Spoor TC, Hammer M, Belloso H: Traumatic hyphema. Failure of steroids to alter its course: a double-blind prospective study. Arch Ophthalmol 1980, 98: 116-9.

12. Kennedy RH and Brubaker RF: Traumatic hyphema in a defined population. Am J Ophthalmol 1988, 106: 123-30.

13. Ng CS, Strong NP, Sparrow JM, Rosenthal AR: Factors related to the incidence of secondary haemorrhage in 462 patients with traumatic hyphaema. Eye (In Press).

14. Rasukin W: Traumatic hyphema. Am J Ophthalmol 1972, 74: 284-92.

15. Edwards WC, Layden WE: Traumatic hyphema. A report of 184 consecutive cases. Am J Ophthalmol 1973, 75: 110-6.

16. Read J and Goldberg MF: Comparison of medical treatment for traumatic hyphema. Trans Am Acad Ophthalmol and Otol 1974, 78: 799-815.

17. Thomas MA, Parrish RK, Feuer WJ: Rebleeding after traumatic hyphema. Arch Ophthalmol 1986, 104: 206-10.

18. Varnek L, Dalsgaard C, Hansen A, Klie F: The effect of tranexamic acid on secondary haemorrhage after traumatic hyphaema. Acta Ophthalmol 1980, 58: 787-93. 